\title{
Modelling of pressure-temperature conditions for cavitation initiation in different liquids
}

\author{
N. Mikhailova ${ }^{1}$, I. Smirnov ${ }^{2}$, G. Volkov ${ }^{3}$ \\ Saint Petersburg University, Universitetskaya nab 7/9, Saint Petersburg, 199034, Russia \\ ${ }^{1}$ Corresponding author \\ E-mail: ${ }^{1}$ n.v.mikhailova@spbu.ru, ${ }^{2}$ i.v.smirnov@spbu.ru, ${ }^{3}$ g.volkov@spbu.ru \\ Received 1 September 2019; accepted 17 September 2019 \\ DOI https://doi.org/10.21595/vp.2019.20983 \\ Check for updates \\ Copyright $(\mathrm{C} 2019$ N. Mikhailova, et al. This is an open access article distributed under the Creative Commons Attribution License, which \\ permits unrestricted use, distribution, and reproduction in any medium, provided the original work is properly cited.
}

\begin{abstract}
The paper presents modelling of acoustic cavitation inception. The model is based on the structural-temporal approach, which has shown a predictive effectiveness in considering various transient processes. The presented calculations were performed for four different liquids at a high-frequency vibration and across a wide temperature range. The predictions of the presented theoretical model were validated by comparison with the experimental data, and good agreement was demonstrated.
\end{abstract}

Keywords: cavitation, structural-temporal approach, incubation time.

\section{Introduction}

Acoustic cavitation has attracted extensive interest for many decades. Studies considering this phenomenon are dedicated to solving a variety of problems in processing applications and chemical reactions. Some have used cavitation as a tool to achieve changes in the properties of liquids, such as grain refinement in different alloying systems [1], viscosity [2] and degassing [3]. The cavitation phenomenon has also been studied extensively. For instance, in [4], a study was made of the bubble shape and the localisation of cavitation. A reduction of cavitation pressure by including impurities was shown in [5], where nanoparticles were dispersed in pure water. Cavitation inception was considered in water with dissolved gases [6], which resulted in decreased liquid strength.

There are many interesting experimental data for this topic. However, development of analytical and numerical models has been slow. The proposed models consider the processes of the development, propagation and interaction of bubbles in a liquid, as well as their collapse and subsequent formation of shock waves or turbulence. At the same time, conditions (criteria) of the initiation, course and outcome of cavitation processes are very difficult to find in the literature. The absence of adequate prognostic models makes it difficult to ascertain the effects of acoustic cavitation and their application in engineering.

This work is devoted to modelling cavitation inception at ultrasound waves. Such models can improve our understanding of liquid strength and the cavitation phenomenon. Results of modelling can be used to determine a proper condition for reducing destructive effects (for example, erosion [7]), allowing us to successfully apply the advantages of cavitation (e.g., filter cleaning [8]). Thus, the cavitation model is a useful tool in solving several engineering problems. In acoustic cavitation, the liquid is filled with vapour bubbles due to the excess negative pressure of the liquid strength, resulting in violation of the integrity. The pressure in the liquid is created by a high-frequency acoustic field, which creates a dynamic loading mode. Therefore, it is proposed that cavitation be considered as a fracture of liquid that allows application of the structural-temporal approach [9] in cavitation modelling. The proposed approach has qualitatively described a number of transient processes [10]. Preliminary studies of the acoustic cavitation model using the proposed approach have shown good agreement with experiments performed in water [11-13].

The purpose of this work is to expand the application of the cavitation model for different 
types of liquid and to determine their strength parameters, which can be applied in further prediction of cavitation for a given loading. The novelty of this approach is its ability to calculate the phase-equilibrium conditions for condensed matters under an ultrasonic field with different parameters. The paper presents the results of simulation of cavitation in water, heavy water, ethanol and heptane. The model with only two parameters allowed us to predict cavitation inception depending on temperature. The theoretical predictions are validated by comparison with experimental data [14].

\section{Analytical model}

Let us consider a theoretical approach for analytical modelling of cavitation initiation. The phenomenon of cavitation is local in nature and arises only in certain circumstances. Therefore, to simplify the model, we assume that the occurrence of cavitation is localised in a constant given region of liquid.

Acoustic cavitation appears in conditions of a sound wave generating such a negative pressure that it results in local boiling and the creation of a gap in the liquid. The pressure, created by a sound wave, can be written as:

$P(t)=A \sin \omega t$,

where $A$ is the amplitude and $\omega$ is the cyclic frequency of the sound wave. Acoustic cavitation is realised in a short time period due to high-frequency loading. For this reason, temporal characteristics should be taken into account in the modelling. We assumed that cavitation initiation occurs during the incubation period $\tau$, and that is in accordance with a criterion based on the structural-temporal approach [10]:

$\frac{1}{\tau(T)} \int_{t^{*}-\tau(T)}^{t^{*}}\left|\frac{P(t)}{P_{c}(T)}\right|^{\alpha} d t>1$,

where $t^{*}$ is time of cavitation inception, and $P_{c}(T)$ is the pressure necessary to generate cavitation during prolonged (static) loading [12] in liquid at temperature $T$. The parameter $\alpha$ depends on viscosity of liquid, and since low-viscosity liquids are considered in this work, the parameter is taken as $\alpha=0.5$ [15]. This pressure is determined by the difference between the external pressure $P_{\text {stat }}$ and the pressure in the liquid-gas phase transition $P_{p h}$ for a given temperature:

$P_{c}(T)=P_{\text {stat }}-P_{p h}(T)$

Since we simulate cavitation across a temperature range, the incubation time $\tau$ also changes due to heating of the liquid, which leads to an increase in the velocity of particles and a decrease in the strength of the liquid. By analogy with the thermo-fluctuation theory, the temperature dependence of the incubation time can be determined as follows:

$\tau(T)=\tau_{0} e^{\frac{G}{k T}}$,

where $G$ is energy needed to start cavitation in an elementary volume, and $\tau_{0}$ is a constant that characterises the strength of liquid at a current scale length.

Cavitation is caused by tensile stresses, and therefore, when substituting the pressure of the sound wave in Eq. (1) into the criterion of Eq. (2), only the range $t \in(0, \pi / \omega)$ was considered. In the model, cavitation pressure is taken as a positive value. Therefore, by replacing $z=\omega t$ and $\lambda(T)=\tau(T) \omega$, we can calculate the pressure $A(T)$ which leads to cavitation: 
$A(T)=\left\{\begin{array}{l}\left.\left.P_{c}(T) \cdot\left|\frac{1}{\lambda(T)} \max _{t_{z} \in(0 ; \pi)} \int_{t_{z}-\lambda(T)}^{t_{z}}\right| \sin z\right|^{\alpha} d z\right|^{-\frac{1}{\alpha}}, \quad \lambda(T) \leq \pi, \\ \left.\left.P_{c}(T) \cdot\left|\frac{1}{\lambda(T)} \int_{0}^{\pi}\right| \sin z\right|^{\alpha} d z\right|^{-\frac{1}{\alpha}}, \quad \lambda(T)>\pi .\end{array}\right.$

Eq. (5) allows one to obtain analytical curves for the dependence of the threshold value of the cavitation pressure on temperature for a given frequency of ultrasonic waves. The frequency and amplitude of ultrasound are determined by the parameters of the ultrasonic transducer, with temperature and pressure also regulated by external conditions. Parameters $\tau_{0}$ and $G$ are determined semi-empirically and depend on the liquid strength and scale length characterised by the bubble size and wave frequency.

\section{Results and discussion}

Modelling of cavitation was carried out for four different fluids: water, heavy water $\left(\mathrm{D}_{2} \mathrm{O}\right)$, heptane and ethanol. The frequency of the ultrasonic field was $1 \mathrm{MHz}$, and the external pressure was about 1.6 MPa. The parameters used to calculate cavitation pressure are presented in Table 1 . The liquid-vapour phase diagrams $\left(P_{p h}(T)\right)$ were taken from the literature $[16,17]$.

Table 1. Modelling parameters

\begin{tabular}{|c|c|c|c|c|}
\hline & Water & Heavy water & Heptane & Ethanol \\
\hline$G, 10^{-23} \mathrm{~J}$ & 400 & 490 & 600 & 450 \\
\hline$\tau_{0}, \mu \mathrm{s}$ & 6.9 & 5.4 & 3.5 & 6 \\
\hline$P_{\text {stat }}, \mathrm{MPa}$ & 1.6 & 1.67 & 1.63 & 1.6 \\
\hline
\end{tabular}
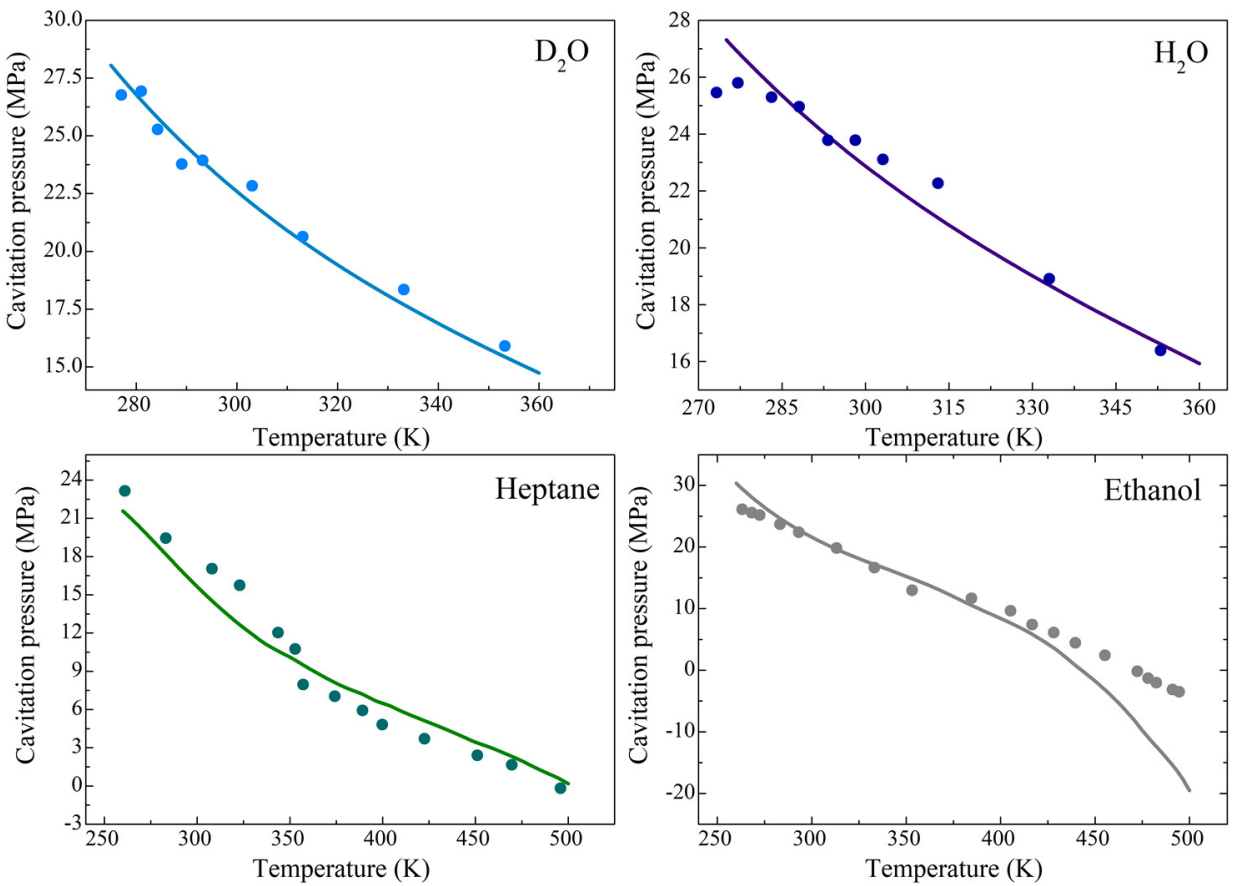

Fig. 1. Cavitation pressure as a function of temperature for heavy water, water, heptane and ethanol. Solid line represents the theoretical calculation; circles represent the experimental data [14]

The modelling results (Fig. 1) show good agreement with the experimental data [14]. The 
authors of [14] use a focused ultrasonic burst and studied the liquid during the period of rarefaction. Only a small volume of bulk liquid was stretched during a short period of time. These conditions make it possible to apply Eq. (5).

Fig. 1 shows that an increase in temperature leads to a reduction in cavitation pressure for all liquids. However, it should be noted that a discrepancy with experimental points is observed for water at low temperatures. It can be explained by the fact that the experiments were performed at a temperature close to the melting point. This can lead to heterogeneity in water, which contributes to bubble formation and further cavitation initiation, resulting in a reduction of cavitation pressure.

A significant discrepancy between the theoretical curve and the experimental data with ethanol occurs when the temperature approaches a critical point $\left(T_{\text {crit }} \cong 514 \mathrm{~K}\right.$ ). The absence of a significant drop in cavitation pressure in the experiments can be the result of features of the liquid properties at ultrahigh temperatures, which the presented model does not consider.

\section{Conclusions}

This paper presents a modelling of cavitation initiation based on the structural-temporal approach, where cavitation is considered as a rupture of the liquid integrity due to its excessive strength. The calculation results showed a good correlation with experiments conducted for four different liquids: water, heavy water, heptane and ethanol. However, the theoretical curves have discrepancies at points that are close to the phase change to a solid state or critical fluid. Thus, avoiding those limits, the presented approach can be easily used in engineering to predict the threshold parameters of cavitation.

\section{Acknowledgement}

This research was supported by the Grant of the President of the Russian Federation for young scientists (MK-2269.2019.8).

\section{References}

[1] Atamanenko T. V., Eskin D. G., Zhang L., Katgerman L. Criteria of grain refinement induced by ultrasonic melt treatment of aluminum alloys containing $\mathrm{Zr}$ and Ti. Metallurgical and Materials Transactions A, Vol. 41, Issue 8, 2010, p. 2056-2066.

[2] Salinas V., Vargas Y., Louisnard O., Gaete L. Influence of the liquid viscosity on the formation of bubble structures in a $20 \mathrm{kHz}$ field. Ultrasonics Sonochemistry, Vol. 22, 2014, p. 227-234.

[3] Rooze J., Rebrov V., Schouten J. C., Keurentjes J. T. F. Dissolved gas and ultrasonic cavitation a review. Ultrasonics Sonochemistry, Vol. 20, 2012, p. 1-11.

[4] Tzanakis I., Lebon G. S. B., Eskin D. G., Pericleous K. A. Characterisation of the ultrasonic acoustic spectrum and pressure field in aluminium melt with an advanced cavitometer. Journal of Materials Processing Technology, Vol. 229, 2016, p. 582-586.

[5] Gu Y., Li B., Chen M. An experimental study on the cavitation of water with effects of $\mathrm{SiO} 2$ nanoparticles. Experimental Thermal and Fluid Science, Vol. 79, 2016, p. 195-201.

[6] Li B., Gu Y., Chen M. An experimental study on the cavitation of water with dissolved gases. Experiments in Fluids, Vol. 58, Issue 12, 2017, p. 164.

[7] Selvam K., Mandal P., Grewal H. S., Arora H. S. Ultrasonic cavitation erosion-corrosion behavior of friction stir processed stainless steel. Ultrasonics Sonochemistry, Vol. 44, 2018, p. 331-339.

[8] Lamminen M. O., Walker H. W., Weavers L. K. Mechanisms and factors influencing the ultrasonic cleaning of particle-fouled ceramic membranes. Journal of Membrane Science, Vol. 237, 2004, p. 213-223.

[9] Petrov Yu, Morozov N. On the modeling of fracture of brittle solids. Journal of Applied Mechanics, Vol. 61, Issue 3, 1994, p. 710-713.

[10] Petrov Yu Incubation time criterion and impulse strength of continuums: fracture, cavitation, electric breakdown. Doklady Akademii Nauk, Vol. 395, Issue 5, 2004, p. 621-625.

[11] Volkov G., Gruzdkov A., Petrov Y. The incubation time criterion and the acoustic strength of sea water. Acoustical Physics, Vol. 53, Issue 2, 2077, p. 119-122. 
[12] Volkov G., Petrov Yu, Gruzdkov A. Acoustic strength of water and effect of ultrasound on the liquidvapor phase diagram. Technical Physics, Vol. 60, Issue 5, 2015, p. 753-756.

[13] Volkov G., Petrov Y., Gruzdkov A. Liquid-vapor phase equilibrium conditions in an ultrasonic field. Doklady Physics, Vol. 60, Issue 5, 2015, p. 229-231.

[14] Arvengas A., Herbert E., Cersoy S., Davitt K., Caupin F. Cavitation in heavy water and other liquids. Journal of Physical Chemistry B, Vol. 115, Issue 48, 2011, p. 14240-14245.

[15] Gruzdkov A. A., Petrov Y. V. Cavitation breakup of low-and high-viscosity liquids. Technical Physics, Vol. 53, Issue 3, 2008, p. 291-295.

[16] Pistorius C., Rapoport E., Clark J. Phase diagrams of $\mathrm{H} 22 \mathrm{O}$ and D2O at high pressures. Journal of Chemical Physics, Vol. 48, Issue 12, 1968, p. 5509-5514.

[17] Phase Behaviour Calculations. Developments in Petroleum Science, Vol. 47, 1998, p. 167-207. 